\section{Detection of retinal} lesions after

\section{telemedicine}

\section{transmission of digital} images
H.L. COOK, G.L. HEACOCK, M.R. STANFORD, J. MARSHALL

\section{Abstract}

Purposelbackground To assess whether loss of image resolution or colour and subsequent telemedicine transmission of digital images affects the accuracy of retinal lesion detection by ophthalmologists when compared with the original transparencies.

Methods Fifteen ophthalmologists of different experience independently scored 11 retinal images for pathological signs. The images were presented as either transparencies or colour and monochrome digital images, which had been transmitted via telephone lines to a geographically remote location. One patient's eye was also imaged using scanning laser ophthalmosocopy (SLO) which produced a dynamic black and white digital image. ANOVA analysis was performed. Results Total scores were higher for transparencies than colour $(p=0.0003)$ or black and white digital images $(p=0.00006)$. Expert observers $(n=5)$ considered separately showed no significant difference of accuracy between transparencies and either colour digital ( $p=0.09$ ) or monochrome digital images $(p=0.11)$. Experts were better than trainees at detecting pathology from less familiar images: total score $(p=0.02)$, colour digital ( $p=0.03)$, monochrome digital $(p=0.02)$ and SLO images $(p=0.004)$.

Conclusion Experienced observers can identify sight-threatening retinal pathology from poorer-resolution digital images that have been transmitted by telemedicine. They can also adapt to viewing less familiar images such as black and white digital or SLO images.

Key words Digital image, Image resolution, Observer accuracy, Retinal lesions, Scanning laser ophthalmoscopy, Telemedicine

Telemedicine is defined as 'the use of telecommunications and information technologies for remote medical care'. ${ }^{1}$ Several studies have assessed telemedicine of radiographic, $^{2-5}$ pathological $^{6}$ and dermatological ${ }^{7}$ images. However, to date little work has been done with transmission of retinal images. ${ }^{8-10}$ There are now several digital image capture devices available for obtaining retinal images, including colour digital fundus cameras $^{11}$ and scanning laser ophthalmoscopes. ${ }^{12}$ However, digital capture produces a much lower resolution than photographic transparencies. The maximum digital image resolution at present is $2500 \times$ 2500 pixels but most commercially available digital fundus camera systems have a maximum of $1200 \times 1000$ pixels or less image. In comparison the maximum resolution of colour transparencies is approximately $3-4$ times greater than the best digital image due to the $1 \mu \mathrm{m}$ grain size and random array of crystals in the film; hence transparencies are currently the standard against which new technologies will be assessed. Further information may be lost from digital images during image compression. To date the level of resolution required for reliable diagnosis of retinal pathology has not been systematically determined.

Attempts have been made to increase the speed of transmission of large-scale data files. This can be done by compressing the image file using techniques such as JPEG; however, this results in loss of data and hence image quality. An alternative method is the use of dedicated digital links such as Integrated Services Digital Network (ISDN) lines. These broadband digital lines can simultaneously transmit voice, video and large data files. As the lines are digital there is no need to convert the original data to an analogue signal for transmission as occurs with transmission via a modem and conventional telephone line, and hence, transmission is much more rapid. Several hospitals now have such lines in place and utilise them for clinical image transmission. However, many primary care centres do not have access to this technology and would rely on standard analogue telephone lines for transmission of images to a hospital centre for expert assessment.
H.L. Cook

G.L Heacock

M.R. Stanford

J. Marshall

Department of

Ophthalmology

GKT Schools

St Thomas' Campus

London, UK

Miss H.L. Cook

Department of

Ophthalmology

GKT Schools

Rayne Institute

St Thomas' Hospital Lambeth Palace Road London SE1 7EH, UK

Tel: +44 (0)1719228110

Fax: +44 (0)171 4019062

e-mail:

HelenLCook@aol.com

Funded by a grant from the National Lottery Charities Fund to the Iris Fund for the Prevention of Blindness

Presented at the Royal College of

Ophthalmologists' Congress meeting in the Fould's Trophy session, 20 May 1999

Received: 8 July 1999 Accepted in revised form: 3 November 1999 


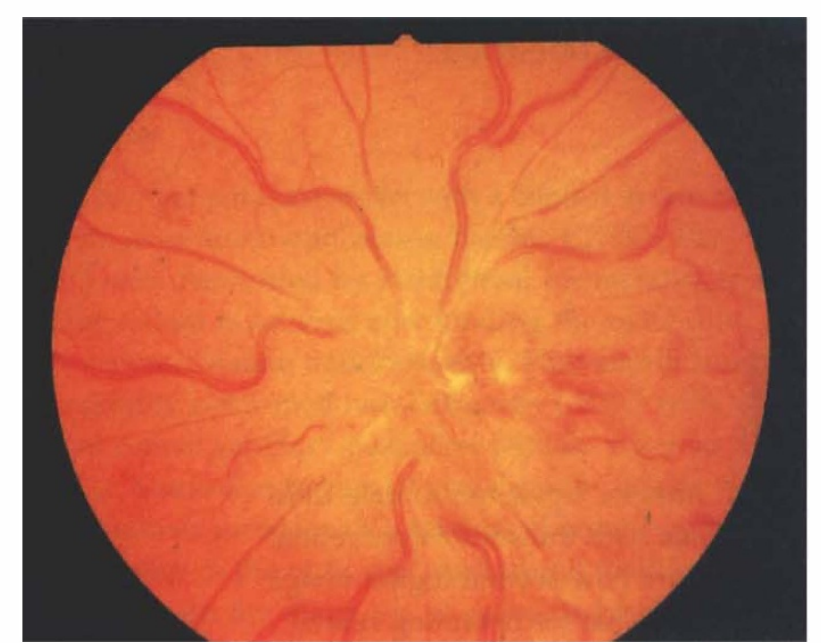

Image 1

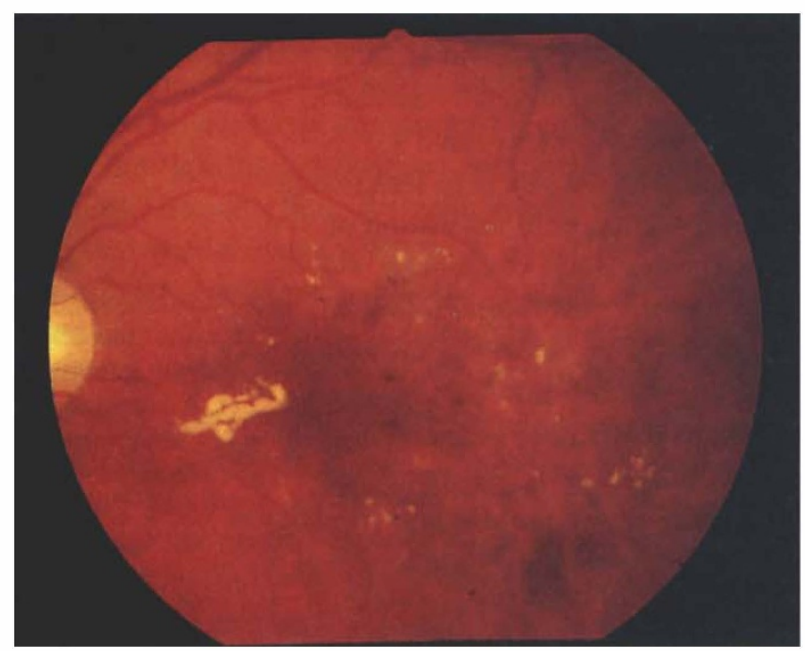

Image 3

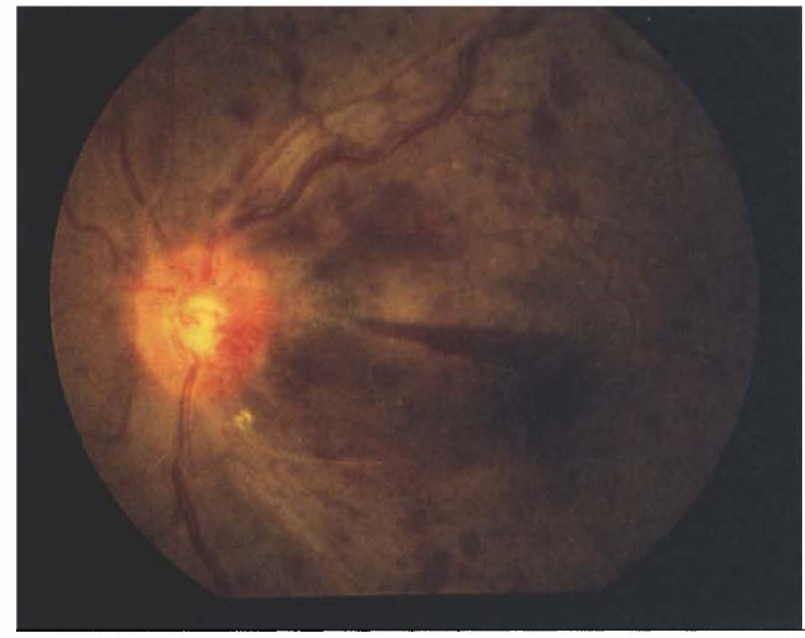

Image 5

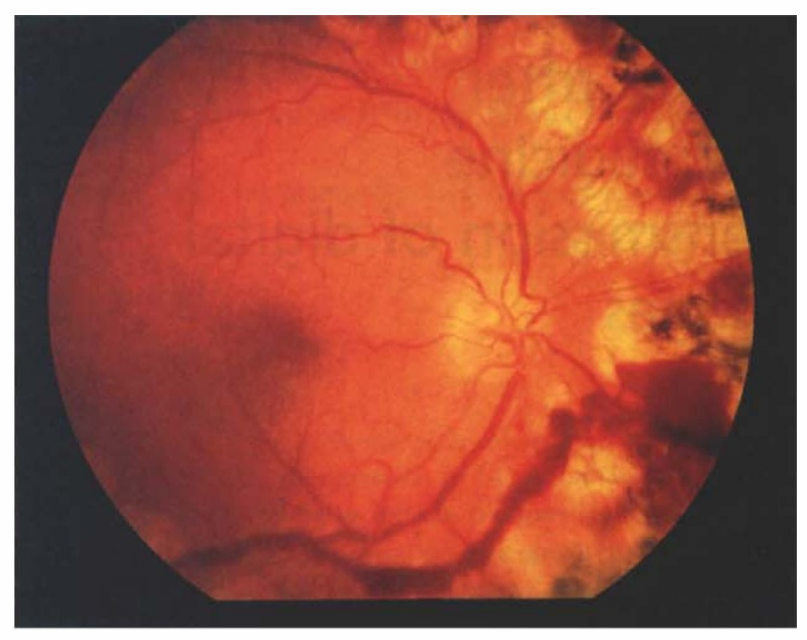

Image 2

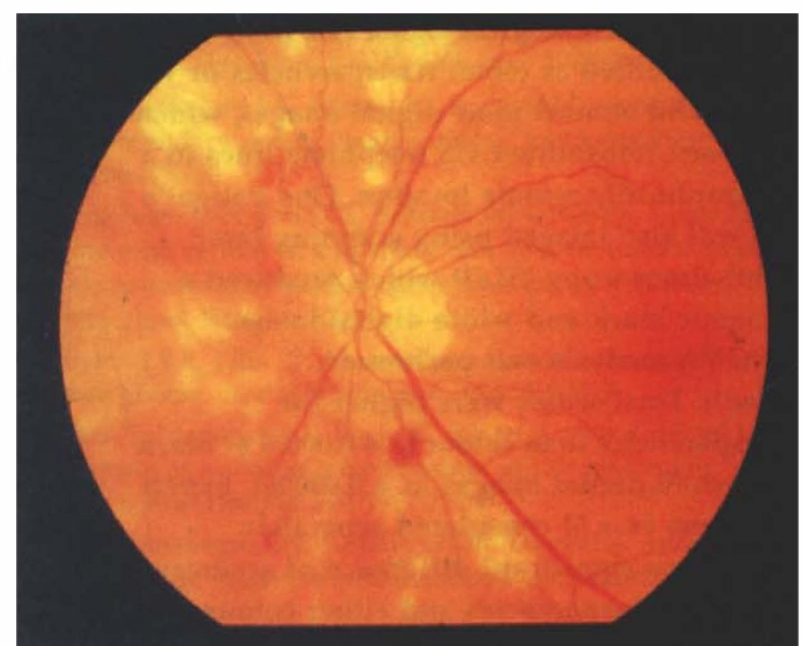

Image 4

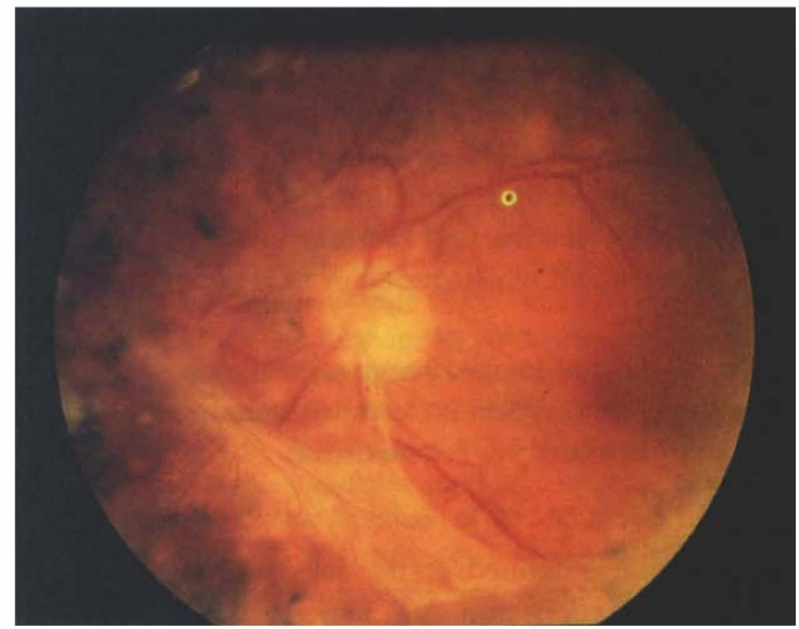

Image 6 


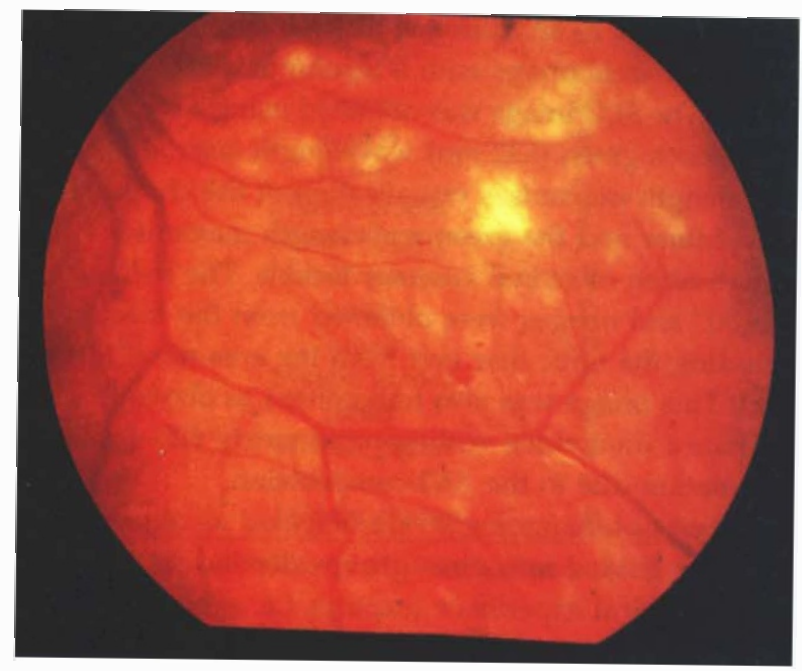

Image 7

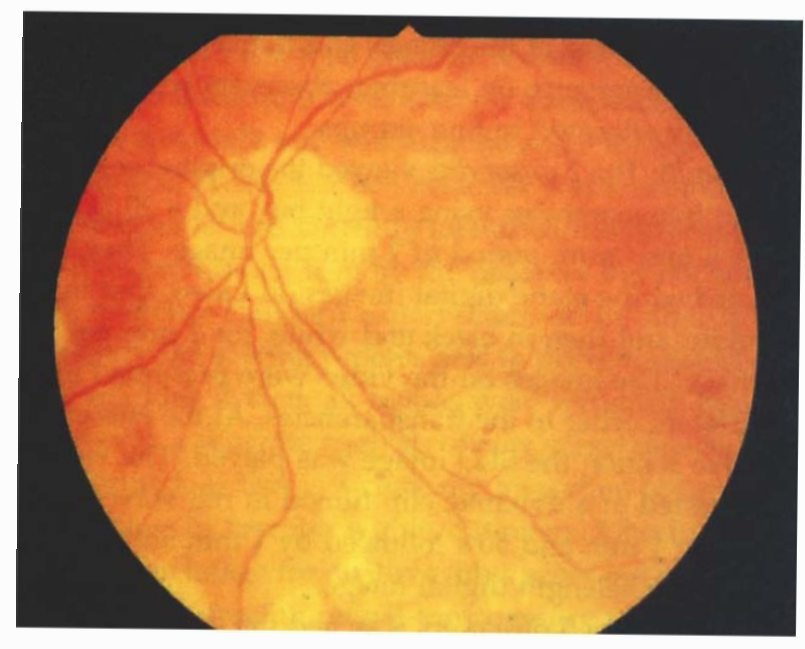

Image 9

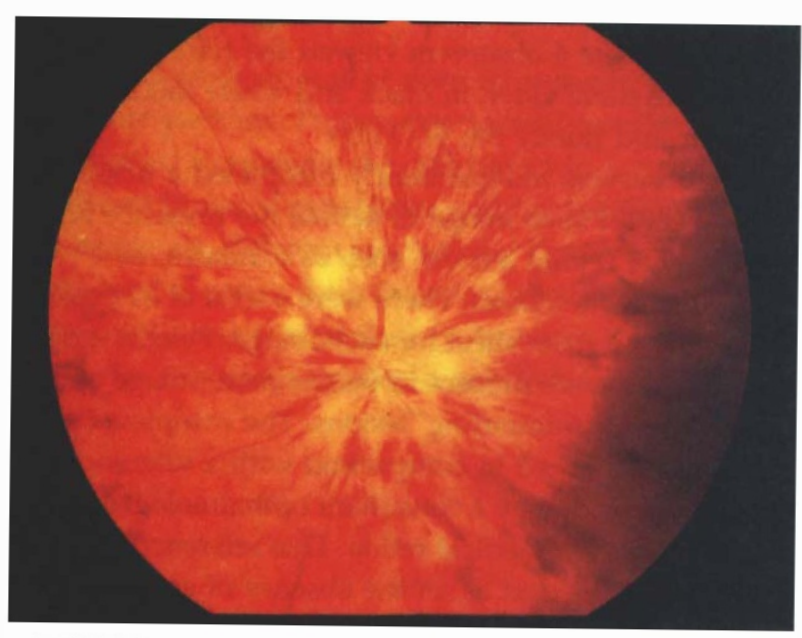

Image 11

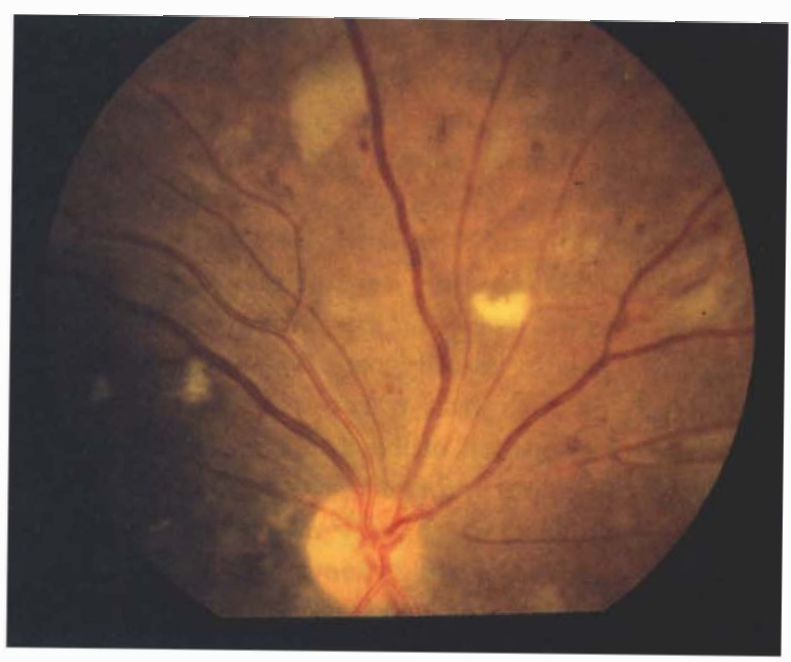

Image 8

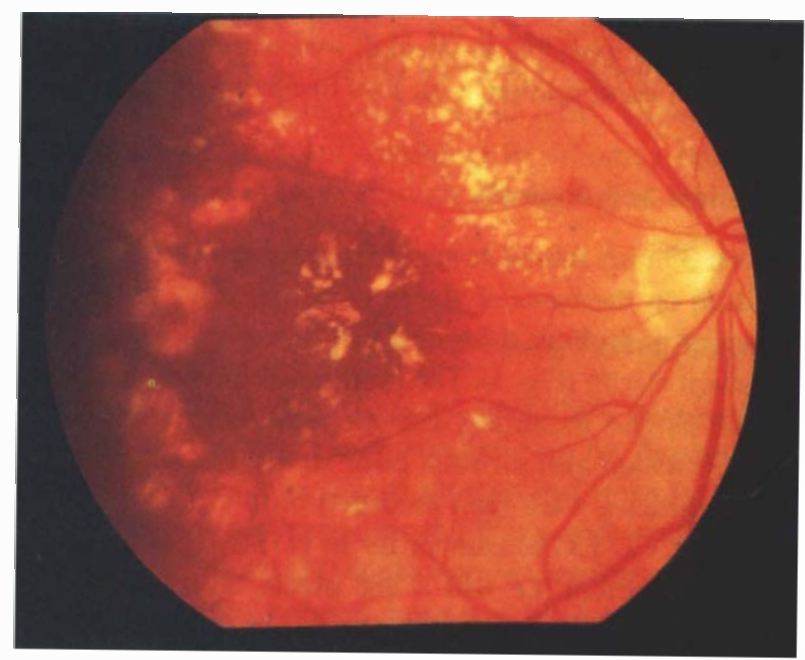

Image 10

Fig. 1. Original colour images used: images 1-11. 
The primary aim of this study was to assess whether digital retinal images transmitted by standard telephone lines are of sufficient quality to detect retinal pathology, in order to simulate a real clinical use of telemedicine.

The secondary aim of this study was to evaluate which element(s) of an image is important for clinical diagnosis of retinal pathology, i.e. the relative importance of resolution, colour and a dynamic image. The study was constructed to evaluate several stages of information loss. The standard used was the ability of ophthalmologists to identify pathological signs from good-quality colour retinal transparencies. We then degraded the resolution of these images by digitising them. Next the colour information was removed to leave a black and white image. Finally we used a lowresolution black and white dynamic image obtained by scanning laser ophthalmoscopy to evaluate the effect of assessing multiple, unfamiliar digital images combined to produce a dynamic image. These images were then transmitted by standard telephone lines and were assessed by ophthalmic clinicians. We also aimed to evaluate the effect of observer expertise to evaluate whether it is possible to de-skill assessment of retinal images in combination with loss of resolution.

\section{Materials and methods}

Eleven $35 \mathrm{~mm}$ retinal transparencies were selected at random on the basis of showing a minimum of five different pathological lesions and being of good to excellent clarity (Fig. 1). The images used were of a range of pathologies including diabetic retinopathy, retinal vein occlusion and swollen optic discs. The selected transparencies were then recorded onto videotape using a Sony \#CCD-FX710 camcorder in NTSC (USA video standard) format (i.e. $640 \times 480$ pixels, 30 frames per second). Video digitisation was used to facilitate direct comparison with the scanning laser ophthalmoscope video segment used in the study. Video images were digitised using codec (compression/decompression) with VideoSpigot software. This programme combines codec and audio video interleaved (AVI) standard to produce digital images with a resolution of $640 \times 480$ pixels with 32 bits of colour information. The images were transmitted from one centre to another geographically distant site via standard telephone lines. Using VideoShare software the received image was transferred to videotape in NTSC (USA video standard) format (i.e. $640 \times 480$ pixels, 30 frames per second). The final video obtained was of 11 static colour digital images. In order to remove colour during the study, the colour on the viewing monitor was turned off. The same monitor was used throughout the experiment with contrast and brightness settings fixed.

One patient whose eye was photographed for the study was also imaged using a prototype portable scanning slit laser ophthalmoscope (SLO) developed in the department. This stream of digital images was included in the study to evaluate the clinical usefulness of lower-quality, dynamic black and white images, as
SLOs may become an important digital image acquisition device in future telemedicine. The SLO video of one eye was recorded as an analogue (RS170) dynamic black and white dynamic image with a resolution of $800 \times 600$ pixels. The SLO image was obtained using a red $(635 \mathrm{~nm})$ and then a green $(532 \mathrm{~nm})$ wavelength laser. The red wavelength maximises visualisation of the choroidal vasculature and the green wavelength maximises visualisation of retinal vascular lesions. The field size was $30^{\circ}$ and images were obtained from the macula including the optic disc and from the area nasal to the disc). This image was also transmitted as above to produce a final video with approximately $60 \%$ loss of information due to the AVI compression.

Fifteen ophthalmologists participated as observers and were graded into three groups depending on their level of clinical experience (experts, i.e. experienced senior registrars or consultants with a special interest in retinal vascular disease; middle graders, i.e. specialist registrars or research registrars; and juniors, i.e. senior house officer level). There were 5 experts, 7 middle graders and 3 juniors. To prevent a learning effect from repeated viewing of the images and to mask the effect of observer experience on learning, observers were then randomly allocated to four groups:

- Group A: The 2 observers viewed the original colour 35 $\mathrm{mm}$ transparencies using a light box and a $20 \mathrm{D}$ lens for a maximum period of $2 \mathrm{~min}$ per image. They later watched the static digital images on video, first in colour and then in black and white, for 2 min per image. The images on the video were presented in a different order to the transparencies. At the end of the video session the SLO image was played. The video consisted of a dynamic clip filmed in red wavelength lasting $1 \mathrm{~min}$ and $50 \mathrm{~s}$ followed by $1 \mathrm{~min}$ and $25 \mathrm{~s}$ of green wavelength digital image.

- Group B: The 5 observers watched the static video in colour first, then in black and white and then the SLO clip. The transparencies were viewed later.

- Group C: The 4 observers scored the static video in black and white, then in colour and then the SLO clip. The transparencies were viewed last.

- Group D: The 4 observers viewed the transparencies, then the static video in black and white followed by colour and finally the SLO clip.

The observers independently completed a tick-box score sheet for each image to denote the presence of any pathological retinal lesions, such as exudates, new vessels or swollen discs. There were a total of 31 possible lesions on the score sheet for each image. Twenty-four of these lesions were present in one or more image and 7 lesions were 'confounding' lesions. The completed score sheets were marked in comparison with a reference standard provided by a consultant opthalmologist with a special interest in medical retina. This reference standard method was selected as, in the absence of a specific quantitative test, the subjective evaluation of retinal appearance by an experienced ophthalmologist is the current basis of clinical diagnosis and treatment of retinal disease. ${ }^{13}$ The reference standard found a total of 62 
Table 1. Total scores of lesions correctly identified

\begin{tabular}{|c|c|c|c|c|c|c|c|}
\hline Observer no. & Experience & Group & Transparencies & Colour digital & $\mathrm{B}+\mathrm{W}$ digital & SLO images & Total \\
\hline 1 & Expert & $\mathrm{D}$ & 62 & 40 & 41 & 8 & 149 \\
\hline 2 & Expert & A & 58 & 49 & 50 & 6 & 163 \\
\hline 3 & Expert & C & 47 & 38 & 36 & 5 & 126 \\
\hline 4 & Expert & B & 39 & 38 & 38 & 4 & 119 \\
\hline 5 & Expert & $\mathrm{C}$ & 46 & 45 & 47 & 4 & 142 \\
\hline 6 & Middle grader & B & 38 & 36 & 31 & 4 & 107 \\
\hline 7 & Middle grader & $\mathrm{D}$ & 42 & 36 & 36 & 2 & 115 \\
\hline 8 & Middle grader & $\mathrm{A}$ & 48 & 43 & 38 & 4 & 133 \\
\hline 9 & Junior & $\mathrm{D}$ & 42 & 35 & 38 & 2 & 116 \\
\hline 10 & Junior & $\mathrm{D}$ & 45 & 31 & 31 & 2 & 108 \\
\hline 11 & Middle grader & $\mathrm{C}$ & 41 & 33 & 29 & 3 & 106 \\
\hline 12 & Junior & B & 29 & 27 & 23 & 2 & 81 \\
\hline 13 & Middle grader & B & 42 & 39 & 39 & 2 & 122 \\
\hline 14 & Middle grader & C & 43 & 31 & 31 & 2 & 107 \\
\hline 15 & Middle grader & B & 44 & 40 & 36 & 4 & 122 \\
\hline
\end{tabular}

$\mathrm{B}+\mathrm{W}$, black and white; SLO, scanning laser ophthalmoscope.

lesions present in the 11 images using the original transparencies. The eye that was also imaged by SLO had 6 lesions when imaged by SLO and 5 when assessed using the transparency alone, as vitreous haemorrhage present in the SLO image was not apparent by photography. The total possible score for the test was 198 , i.e. a maximum of 62 each for transparencies, colour digital and black and white digital and 6 for red SLO and green SLO (Table 1). Each observer was scored for number of lesions correctly identified, lesions missed and 'extra' lesions seen by the observer but not seen by the reference standard expert observer. In order to simplify the scoring process and to prevent distortion of the marking system all lesions were weighted equally; however, a separate analysis of new vessel detection was performed, as this is a clinically important lesion regardless of location within the retina. Observers also scored the image for quality with a score out of 10 , where 10 is a high-quality retinal transparency viewed on a light box.

\section{Statistical analysis}

Statistical analysis was performed using SPSS 7.51 software package. Paired, two-tailed $t$-tests were performed to evaluate the differences in accuracy for the different levels of experience and for the different types of image, i.e. different resolutions. Two-way ANOVA was used to assess the influence of the learning effect. An unpaired $t$-test was performed to assess the perceived difference of image quality scores between the different media.

\section{Results}

The maximum possible score for the test was 198. The mean score for the test for all observers was 121.07 (SD 20.02).

\section{Transparency versus colour digital image}

The score obtained for the colour digital images was compared with the score for the transparencies in order to assess the effect of maintaining colour but reducing resolution. The mean score for transparencies was 44.4 (SD 7.82) out of a possible score of 62. Mean score for colour digital images was 37.4 (SD 5.73). There was a statistically significant difference in accuracy rates for transparencies compared with colour digital images $(p=0.0003)$.

\section{Transparency versus black and white digital image}

To assess the effect of reduced resolution plus loss of colour, comparison was made between the overall scores for black and white digital images and transparencies. The overall mean score for the black and white digital images was 36.26 (SD 6.84) from a possible score of 62 . Hence, for all observers there was a significant increase in accuracy rates for transparencies compared with black and white digital images $(p=0.00006)$.

\section{Colour versus black and white digital image}

Assessing the accuracy scores of the telemedicine digital images in colour and black and white, i.e. images of the same pixel resolution but with colour removed, there was no statistically significant difference between scores when all observers were considered $(p=0.12)$.

\section{Transparency versus dynamic black and white digital image}

To evaluate the impact of a dynamic element in an image with reduced resolution and no colour, the scores for the SLO video were compared with the scores for the transparency of the same eye. From a possible total score of 5 for the transparency of image 4, the overall mean score was 3.06 (SD 1.33). A total score of 6 was possible with SLO imaging as vitreous haemorrhage present could be identified using the SLO but was not apparent on the transparency taken the same day as the SLO 
Table 2. Total scores of 'extra' lesions, i.e. lesions seen by the observer but not present in the definitive diagnosis by the reference standard observer

\begin{tabular}{|c|c|c|c|c|c|c|c|}
\hline Observer no. & Experience & Group & Transparencies & Colour digital & $\mathrm{B}+\mathrm{W}$ digital & SLO images & Total \\
\hline 1 & Expert & $\mathrm{D}$ & 0 & 12 & 18 & 3 & 30 \\
\hline 2 & Expert & A & 2 & 11 & 13 & 0 & 24 \\
\hline 3 & Expert & $\mathrm{C}$ & 10 & 9 & 11 & 0 & 30 \\
\hline 4 & Expert & $\mathrm{B}$ & 4 & 7 & 6 & 0 & 17 \\
\hline 5 & Expert & $\mathrm{C}$ & 21 & 20 & 17 & 1 & 59 \\
\hline 6 & Middle grader & B & 14 & 20 & 15 & 0 & 50 \\
\hline 7 & Middle grader & $\mathrm{D}$ & 11 & 7 & 6 & 1 & 25 \\
\hline 8 & Middle grader & A & 26 & 25 & 19 & 1 & 71 \\
\hline 9 & Junior & $\mathrm{D}$ & 16 & 6 & 8 & 6 & 33 \\
\hline 10 & Junior & $\mathrm{D}$ & 21 & 11 & 8 & 0 & 38 \\
\hline 11 & Middle grader & $\mathrm{C}$ & 12 & 9 & 9 & 2 & 33 \\
\hline 12 & Junior & $\mathrm{B}$ & 9 & 7 & 11 & 2 & 19 \\
\hline 13 & Middle grader & $\mathrm{B}$ & 8 & 13 & 11 & 3 & 35 \\
\hline 14 & Middle grader & C & 15 & 18 & 14 & 2 & 48 \\
\hline 15 & Middle grader & B & 19 & 23 & 27 & 2 & 60 \\
\hline
\end{tabular}

$\mathrm{B}+\mathrm{W}$, black and white; SLO, scanning laser ophthalmoscope.

image. The mean score for all observers viewing the same eye imaged by SLO in red wavelength laser was 1.6 (SD 0.82) from a possible score of 6 . For green wavelength imaging the mean score was 1.87 (SD 1.06). There was no significant difference between the scores obtained with red or green SLO imaging $(p=0.33)$. There was a significant difference between the scores for image 4 obtained by transparency compared with SLO red ( $p=$ $0.0006)$ and SLO green $(p=0.0007)$.

\section{Effect of experience}

The mean total score for lesions correctly identified by expert observers was 139.8 (range 119-163, SD 17.68), by middle grade ophthalmologists was 116.0 (range 106-133, SD 10.20) and by juniors was 101.7 (range 81-116, SD 13.34). Using one-way ANOVA there was a statistically significant difference between the total score obtained by experts and non-experts $(p=0.02)$. There was no statistically significant difference in the accuracy levels of experts versus juniors when identifying lesions from transparencies $(p=0.07)$. However, when grading lesions from lower-resolution images, experts were statistically significantly better than trainees at correctly identifying lesions ( $p=0.03$ for colour digital, $p=0.02$ for black and white digital and $p=0.004$ for SLO images). There was no significant difference in the number of 'extra' lesions seen by the observers when comparing experts against more junior clinicians (Table 2).

As the experts were significantly better at identifying lesions from lower-resolution images than juniors, analysis of the effect of reduced resolution and loss of colour was performed using the scores obtained by the expert group only. Comparing colour digital with transparencies there was no significant difference $(p=$ 0.09). This was also the case when comparing black and white digital against transparencies $(p=0.11)$. Assessing the accuracy scores of the telemedicine digital images in colour and black and white, i.e. images of the same pixel resolution but with colour removed, there was no statistically significant difference between scores when experts alone were considered $(p=0.59)$. Comparing the scores obtained by experts only using the SLO images versus the transparency of image 4 there was a significant difference for the scores obtained $(p=0.03)$.

\section{Learning effect}

The order of viewing the images had no effect on the total score $(p=0.16)$ when all observers were considered. This effect remained non-significant when each medium was considered individually ( $p=0.09$ for transparencies, $p=0.14$ for colour digital, $p=0.35$ for black and white digital and $p=0.72$ for SLO).

Two-way ANOVA was used to assess the effect of experience and take into account the order of viewing the images, i.e. whether watching the digital images before viewing the transparencies affected the final score. This demonstrated that the effect of experience on the final scores was significant $(p=0.003)$ regardless of order of viewing. This effect was true when each type of presentation of the images was considered $(p=0.01$ for transparencies, $p=0.01$ for colour digital, $p=0.046$ for black and white digital and $p=0.03$ for SLO).

\section{Identification of new vessels}

Five images demonstrated six episodes of new vessels (one eye had new vessels on the disc and elsewhere). This gave a total possible score of 20 episodes of new vessels identifiable throughout the test. For all observers there was a significant improvement in detection of new vessels using transparencies compared with colour $(p=$ $0.001)$ and black and white digital images $\left(p<10^{-6}\right)$ and when comparing colour with black and white digital images $(p=0.04)$.

When the expert observers' scores were analysed alone (Table 3) the overall mean score for all methods of viewing the images was 7.6 (SD 2.61). If new vessels classified as intraretinal microvascular abnormalities were included, the total mean score for experts rose to 10 (SD 1.32). The mean score for identification from transparencies was 3.4 (SD 2.30). The mean scores for colour and black and white digital images were 
Table 3. Identification of new vessels by the five expert observers

\begin{tabular}{|c|c|c|c|c|c|c|c|c|c|}
\hline \multirow{2}{*}{$\begin{array}{c}\text { Observer } \\
\text { no. }\end{array}$} & \multicolumn{2}{|c|}{ Transparencies } & \multicolumn{2}{|c|}{ Colour digital } & \multicolumn{2}{|c|}{$\mathrm{B}+\mathrm{W}$ digital } & \multicolumn{2}{|c|}{ SLO images } & \multirow[b]{2}{*}{ Total } \\
\hline & Actual & Possible & Actual & Possible & Actual & Possible & Actual & Possible & \\
\hline 1 & 6 & 6 & 0 & 6 & 1 & 6 & 1 & 2 & 8 \\
\hline 2 & 5 & 6 & 1 & 6 & 3 & 6 & 1 & 2 & 10 \\
\hline 3 & 3 & 6 & 2 & 6 & 0 & 6 & 1 & 2 & 6 \\
\hline 4 & 0 & 6 & 2 & 6 & 1 & 6 & 1 & 2 & 4 \\
\hline 5 & 3 & 6 & 4 & 6 & 2 & 6 & 1 & 2 & 10 \\
\hline
\end{tabular}

$\mathrm{B}+\mathrm{W}$, black and white; SLO, scanning laser ophthalmoscope.

respectively 1.8 (SD 1.48) and 1.2 (SD 1.14). All five experts identified new vessels in the green wavelength SLO images, which optimises imaging of vascular structures.

Comparing the ability of experts to identify new vessels in unfamiliar lower-resolution media, there was no significant difference between transparencies and colour digital $(p=0.07)$ and SLO using the green wavelength $(p=0.37)$. There was, however, a significantly poorer ability to identify neovascularisation using black and white still digital images $(p=0.02)$.

\section{Subjective image quality}

Assessing the mean quality scores assigned, the mean image quality for transparencies was 8.81 (SD 0.91), for colour digital was 6.70 (SD 1.26), for black and white digital was 5.45 (SD 1.74) and for SLO images was 2.75 (SD 1.27). There was a statistically significant difference between the subjective quality scores for the transparencies and the other media and between the colour and black and white digital images $(p<0.05$ for all).

\section{Discussion}

The current standard in retinal imaging remains $35 \mathrm{~mm}$ transparencies. The persistence of this standard in the era of digital imaging is due to the superior image resolution of photographic film, the true colour and the familiarity of the images. The aim of this study was to systematically evaluate the perceived problems of digital imaging by assessing the effect on observer accuracy of reducing resolution, subtracting colour and introducing a dynamic element to the image. It was shown that considering observers of all levels of experience, higher scores were obtained for transparencies than when evaluating images with a poorer resolution. However, the expert observers proved to be considerably more accurate at detecting retinal lesions than less experienced observers, both for transparencies and images of poorer resolution.

When expert observers were considered alone, there was no statistically significant difference between scores obtained for the colour transparencies and poorerresolution images (colour digital), images with reduced resolution and loss of colour information (black and white digital) and between images of the same resolution with and without colour. However, there was a significant difference between the scores for transparencies and unfamiliar, dynamic images of poorer resolution and no colour produced by a prototype lowcost portable scanning laser ophthalmoscope, although it is likely that with increasing familiarity, accuracy would increase.

\section{Reduction of resolution}

The ability to diagnose pathological lesions accurately from digital images, when compared with original film, has been demonstrated in several studies assessing radiological images. ${ }^{14-19}$ It is known that diagnostic accuracy for $\mathrm{X}$-ray images increases with increasing pixel number or decreasing pixel size. ${ }^{19}$ To our knowledge there have been no studies evaluating the effect of reduction of resolution on the diagnostic accuracy of retinal images.

\section{Loss of colour information}

In this study there was no difference in accuracy levels of observers between colour and grey-scale images of the same resolution. It has been demonstrated that only a limited amount of colour information is required to diagnose pathology in the case of endoscopic ${ }^{21}$ and histological $^{22}$ lesions. These studies assessed the effect of reducing colour information in digital images by reducing the digital colour information from true colour (24-bit, i.e. 8 bits each for red, blue and green, giving a palette of 16.7 million colours) to 8-bit (a palette of 256 colours). It was found in both studies that diagnostic accuracy was not affected by reducing the colour information to 8 bits.

A study evaluating grey-scale image telepathology of frozen sections showed adequate diagnostic accuracy despite the lack of colour. ${ }^{23}$ Similarly, a study using black and white $35 \mathrm{~mm}$ transparencies to diagnose diabetic retinopathy found that the diagnosis of level of retinopathy present was not impaired by the lack of red, green and blue input. ${ }^{24}$ Ophthalmologists are also familiar with evaluating black and white images produced by fluorescein angiography, although contrast in these images is maximised due to the contrast medium used.

Radiologists who rely on grey-scale images for all diagnoses have set standards ${ }^{25}$ with a minimum requirement of $2000 \times 2000$ pixels and 10 bits of grey level for the analysis of plain radiographs. It has been suggested that for pathology the spatial resolution 
required is somewhere in the region of $1000 \times 1000$ pixels. ${ }^{26}$ However, the types of images viewed are very different in endoscopy, pathology and radiology compared with ophthalmology, and this tissue has not been addressed for retinal images.

\section{Scanning laser ophthalmoscope images}

The SLO images obtained were of reduced resolution and had no colour information. The red image was more difficult to interpret as there is greater visualisation of the choroidal vasculature than is seen by conventional white light viewing of the retina. The green wavelength image had increased contrast of retinal vascular structures and hence was more similar to traditional retinal photographic images. The images were dynamic, which may have facilitated detection of vitreous haemorrhage and the mobile disc new vessels present. Studies comparing dynamic versus static image assessment in pathology suggest that there is a higher accuracy with dynamic images. ${ }^{27,28}$ This is likely to be due to a wider range of fields being available in dynamic imaging, which increases the amount of information available to the observer to facilitate decision making, as occurs with slit-lamp biomicroscopy examination of the fundus.

The transmitted SLO image used in this study had an approximately $60 \%$ reduction in resolution compared with the original SLO image. This was perceived by the observers as a subjective reduction in image quality when compared with the digital colour and black and white images. However, new vessels and vitreous haemorrhage were identified by most observers from the green wavelength SLO image due to the selective enhancement of these lesions.

\section{Observer experience}

Clinical diagnosis is a complex process involving evaluation of various components in an image and comparing them with the cortical database of the observer. It is also a highly subjective process and it is not surprising that the scores within each experience level were fairly widely distributed. However, more experienced observers were able to extract more information from poorer-quality images than were less experienced clinicians. This was confirmed for all media except $35 \mathrm{~mm}$ transparencies, where there was no statistically significant difference between expert observers and junior ophthalmologists. This implies that experts are better at extracting useful information from a retinal image, despite the image being in an unfamiliar format such as black and white still or dynamic digital images.

In telepathology, it has been demonstrated that observers who have experience of viewing video monitors, such as computers screens, perform better. ${ }^{29-33}$ It could be assumed from this that younger observers, who are likely to be more familiar with video and computer games, would be at an advantage. This factor may have influenced the performance of some observers in this study but was not specifically assessed. From our results, clinical experience was strongly correlated with accuracy.

\section{Detection of new vessels}

The detection of new vessels is an important clinical sign and therefore this was assessed specifically. For expert observers there was no significant reduction in the ability to detect new vessels from colour digital or SLO images compared with transparencies. However, the detection rate was significantly poorer using still black and white digital images. This may be due to the narrow range of grey scale, which produced poorer contrast between new vessels and the background retinal tissue. The dynamic green wavelength SLO image demonstrated the mobile nature of the new vessels present, which facilitated detection and also used wavelength-specific monochromatic light to improve contrast.

\section{Clinical applications of telemedicine}

Diabetic retinopathy screening is one clinical situation where telemedicine could prove to be a cost-effective use of technology. There are fewer than 2000 ophthalmologists of all grades in the UK and in theory this group should review approximately 2 million diabetics every year. ${ }^{34}$ The best way to assess a patient is for a specialist to examine the patient. However, in many situations it is impractical for this to occur, due to poor mobility of the patient, geographical distance, limited specialist time and cost. Therefore, use of $35 \mathrm{~mm}$ transparencies viewed using a light box and hand-held $20 \mathrm{D}$ lens is now an accepted method of screening. ${ }^{35-37}$ A further application would be optic disc evaluation in glaucoma screening.

There are two levels of screening. Level 1 screening is the simple determination of whether the image is normal or abnormal. Work is currently under way in a number of centres to use neural networks to automatically evaluate and sort images into normal and abnormal at the time of screening. The second level of screening determines whether the abnormality is clinically significant and requires further expert clinical evaluation and treatment. Therefore screening systems could be refined and become less labour-intensive if only abnormal images were transmitted for a level 2 expert evaluation. This study demonstrates that it is feasible to transmit retinal images via a telemedicine link and still identify important pathological lesions, provided the observer is clinically experienced. However, further studies are required to establish the standards required for image resolution and quality before telemedicine of retinal images is widely adopted in clinical practice. This study deliberately used standard telephone lines in order to assess the 'real-life' clinical situations where telemedicine is likely to be useful, i.e. transmission of images from a primary to secondary care using existing 
communication links in order to minimise costs. This was possible despite the loss of resolution due to image compression necessary to facilitate image transfer.

\section{References}

1. Perednia DA, Allen A. Telemedicine technology and clinical applications. JAMA 1995;273:483-8.

2. Yoshino MT, Carmody R, Fajardo LL, Seeger J, Jones K. Diagnostic performance of teleradiology in cervical spine fracture detection. Invest Radiol 1992;27:55-9.

3. Scott WW, Rosenbaum JE, Ackerman SJ, Reichle RL, Magid D, Weller JC, et al. Subtle orthopaedic fractures: teleradiology workstation versus film interpretation. Radiology 1993;187:811-5.

4. Goldberg MA, Rosenthal DI, Chew FS, Blickman JG, Miller SW, Mueller PR. New high-resolution teleradiology system: prospective study of diagnostic accuracy in 685 transmitted clinical cases. Radiology 1993;186:429-34.

5. Andersen EB, Borgesen SE, Jensen KK, Kruse T, Rahbek M. Image transmission between hospitals via the ordinary telephone network. Ugeskrift for Laeger 1995;157:2155-8.

6. Weinberg DS, Allaert FA, Dusserre P, Drouot F, Retailliau B, Welch WR, et al. Telepathology diagnosis by means of digital still images: an international validation study. Hum Pathol 1996;27:111-8.

7. Kvedar JC, Edwards RA, Menn ER, Mofid M, Gonzalez E, Dover J, et al. The substitution of digital images for dermatologic physical examination. Arch Dermatol 1997;133:161-7.

8. Loshin DS, Caputo MP. Application of telemedicine in the diagnosis of posterior fundus ocular disease. Invest Ophthalmol Vis Sci 1995;36:S429.

9. Li HK, Tang RA, Schiffman JS, Sy RT, Temprano JE, Prager TC. Diagnosing cytomegalovirus retinitis through telemedicine in a Texas correctional institution: a pilot study. Invest Ophthalmol Vis Sci 1996;37:S898.

10. Laird M, Deen M, Brooks S, Threlkeid A, Hull D, Shatz A, et al. Telemedicine diagnosis of diabetic retinopathy, cataract and glaucoma by direct ophthalmoscopy. Invest Ophthalmol Vis Sci 1996;37:492.

11. Friberg TR, Rehkopf PG, Warnicki JW, Eller AW. Use of directly acquired digital fundus and fluorescein angiography images in the diagnosis of retinal disease. Retina 1987;7:246-51.

12. Webb RH, Hughes GW, Pomerantzeff O. Flying spot TV ophthalmoscope. Appl Optics 1980;19:2991-7.

13. Blumberg MS. Evaluating health screening procedures. Oper Res 1957;5:351-60.

14. Goodman LR, Foley WD, Wilson CR, Rimm AA, Lawson TL. Digital and conventional chest images: observer performance with film digital radiography system. Radiology 1986;158:27-33.

15. Chakraborty DP, Breatnach ES, Yester MV, Soto B, Barnes GT, Fraser RG. Digital and conventional chest imaging: a modified ROC study of observer performance using simulated nodules. Radiology 1986;158:35-9.

16. MacMahon H, Metz CE, Doi K, Kim T, Giger ML, Chan H-P. Digital chest radiography: effect on diagnostic accuracy of hard copy, conventional video and reversed gray scale video display formats. Radiology 1988;168:669-73.

17. Hayrapetian A, Aberle DR, Huang HK, Fiske R, Morioka C, Valentino D, et al. Comparison of 2048-line digital display formats and conventional radiographs: an ROC study. AJR 1989;152:1113-8.
18. Wegryn SA, Piraino DW, Richmond BJ, Schluchter MD, Uetani M, Freed HA, et al. Comparison of digital and conventional musculoskeletal radiography: an observer performance study. Radiology 1990;175:225-8.

19. Cox GG, Cook LT, McMillan JH, Rosenthal SJ, Dwyer SJ. Chest radiography: comparison of high-resolution digital displays with conventional and digital film. Radiology 1990;176:771-6.

20. MacMahon H, Vyborny CJ, Metz CE, Doi K, Sabetti V, Solomon SL. Digital radiography of subtle pulmonary abnormalities: an ROC study of the effect of pixel size on observer performance. Radiology 1986;158:21-6.

21. Vakil N, Bougeois K. A prospective, controlled trial of eightbit, 16-bit and 24-bit digital color images in electronic endoscopy. Endoscopy 1995;27:589-92.

22. Doolittle MH, Doolittle KW, Winkelman Z, Weinberg DS. Color images in telepathology: how many colors do we need? Hum Pathol 1997;28:36-41.

23. Oberholzer M, Fischer H-R, Christen H, Gerber S, Bruhlmann M, Mihatsch M, et al. Telepathology with an integrated services digital network: a new tool for image transfer in surgical pathology. A preliminary report. Hum Pathol 1993;24:1078-85.

24. de Sonnaville JJJ, van der Feltz van der Sloot D, Ernst L, Wijkel D, Heine RJ. Retinopathy screening in type 2 diabetes: reliability of wide angle fundus photography. Diabetic Med 1996;13:482-6.

25. Reston VA. ACR standard for teleradiology. American College of Radiology, 1994.

26. Black-Schaffer S, Flotte TJ. Current issues in telepathology. Telemed 1995;1:95-106.

27. Shimosato Y, Yagi Y, Yamagishi K, Mukai K, Hirohashi S, Matsumoto T, et al. Experience and present status of telepathology in the National Cancer Center Hospital, Tokyo. Zentralb Pathol 1992;138:413-7.

28. Perednia DA, Gaines JA, Butruille TW. Comparison of the clinical informativeness of photographs and digital imaging media with multiple-choice receiver operating characteristic analysis. Arch Dermatol 1995;131:292-7.

29. Weinstein RS, Bloom KJ, Rozek LS. Telepathology and the networking of pathology diagnostic services. Arch Pathol Lab Med 1987;111:646-52.

30. Bloom KJ, Rozek SL, Weinstein RS. ROC curve analysis of super high resolution video for histopathology. SPIE Proc Visual Commun Image Processing 1987;845:408-12.

31. Weinstein RS, Bloom KJ, Krupinski EA, Rozek LS. Human performance studies of the video microscopy component of a dynamic telepathology system. Zentralbl Pathol 1992;138:399-401.

32. Krupinski EA, Weinstein RS, Bloom KJ, et al. Progress in telepathology: system implementation and testing. Adv Pathol Lab Med 1993;6:63-87.

33. Krupinski EA, Weinstein RS, Rozek SL. Experience-related differences in diagnosis from medical images displayed on monitors. Telemed J 1996;2:101-8.

34. Amos AF, McCarty DJ, Zimmet P. The rising global burden of diabetes and its complications: estimates and projections to the year 2010. Diabetic Med 1997;14:S7-S85.

35. Retinopathy Working Party. A protocol for screening for diabetic retinopathy in Europe. Diabetic Med 1991;8:263-7.

36. Anonymous. Screening guidelines for diabetic retinopathy. American College of Physicians, American Diabetes Association and American Academy of Ophthalmology. Ann Intern Med 1992;116:683-5.

37. British Diabetic Association. Retinal photography screening for diabetic eye disease. London: British Diabetic Association, 1997. 